Applied Physiology, Nutrition, and Metabolism

Canadian Science Publishing Physiologie appliquée, nutrition et métabolisme

\title{
Efficacy of downhill running training for improving muscular and aerobic performances
}

\begin{tabular}{|r|l|}
\hline Journal: & Applied Physiology, Nutrition, and Metabolism \\
\hline Manuscript ID & apnm-2017-0538.R1 \\
\hline Manuscript Type: & Article \\
\hline Date Submitted by the Author: & 25-Oct-2017 \\
\hline Complete List of Authors: & $\begin{array}{l}\text { Toyomura, Junichi; National Institute of Fitness and Sports in Kanoya } \\
\text { Mori, Hisashi; Ritsumeikan University } \\
\text { Tayashiki, Kota; National Institute of Fitness and Sports in Kanoya } \\
\text { Yamamoto, Masayoshi; National Institute of Fitness and Sports in Kanoya, } \\
\text { Sports and Life Science } \\
\text { Kanehisa, Hiroaki; National Institute of Fitness and Sports in Kanoya, } \\
\text { Maeo, Sumiaki; Waseda University, Faculty of Sport Sciences }\end{array}$ \\
\hline $\begin{array}{r}\text { Is the invited manuscript for } \\
\text { consideration in a Special } \\
\text { Issue? : }\end{array}$ & $\begin{array}{l}\text { knee extension strength, change-of-direction ability, aerobic capacity, } \\
\text { heart rate-matched running, eccentric component }\end{array}$ \\
\hline Keyword: & \\
\hline \multicolumn{2}{|c|}{}
\end{tabular}

\section{SCHOLARONE}

Manuscripts 


\title{
Efficacy of downhill running training for improving muscular
}

\section{and aerobic performances}

\author{
Junichi Toyomura ${ }^{1,2}$, Hisashi Mori ${ }^{3,4}$, Kota Tayashiki ${ }^{1}$, Masayoshi Yamamoto ${ }^{1}$, \\ Hiroaki Kanehisa $^{1}$, Sumiaki Maeo ${ }^{4,5,6}$
}

${ }^{1}$ Department of Sports and Life Sciences, National Institute of Fitness and Sports in Kanoya, 1 Shiromizu, Kanoya, Kagoshima, 891-2393, Japan

${ }^{2}$ Miura Dolphins Co. Ltd., 3-10-3 Sendagaya, Shibuya, Tokyo, 151-0051, Japan

${ }^{3}$ Research Organization of Science and Technology, Ritsumeikan University, 1-1-1 Nojo-Higashi, Kusatsu, Shiga, 525-8577, Japan

${ }^{4}$ Japan Society for the Promotion of Science, 5-3-1 Koujimachi, Chiyoda, Tokyo, 102-0088, Japan

${ }^{5}$ Faculty of Sport Sciences, Waseda University, 2-579-15 Mikajima, Tokorozawa, Saitama, 359-1192, Japan

${ }^{6}$ School of Sport, Exercise and Health Sciences, Loughborough University, Loughborough, Leicestershire, LE11 3TU, United Kingdom

\section{Corresponding author: Sumiaki Maeo}

E-mail: smaeo1985@gmail.com

Telephone: $+81-429-476945$

E-mail address: Junichi Toyomura: toyomura@snowdolphins.com, Hisashi Mori: m137009@gst.ritsumei.ac.jp, Kota Tayashiki: m157005@sky.nifs-k.ac.jp, Masayoshi Yamamoto: yamamoto@nifs-k.ac.jp, Hiroaki Kanehisa: hkane@nifs-k.ac.jp 


\section{Abstract}

This study investigated effects of downhill (DR) versus level (LR) running training on various muscular and aerobic performances. Eighteen healthy young males conducted either DR (DR group [DRG], $\mathrm{n}=10:-10 \%$ slope) or LR (LR group [LRG], $\mathrm{n}=8$ ) training at a target heart rate (HR) associated with lactate threshold (LT) for $20 \mathrm{~min} \cdot \mathrm{session}^{-1}, 3$ sessions $\cdot$ week $^{-1}$, for 5 weeks. Before and after the interventions, the following variables were measured: knee extension torque $\left(-150,-30,0,30,150^{\circ} \cdot \mathrm{s}^{-1}\right)$, leg extension power (simultaneous hip and knee extension: $0.8 \mathrm{~m} \cdot \mathrm{s}^{-1}$ ), squat and counter-movement jump height, rebound jump index (jump height $\cdot$ contact time ${ }^{-1}$ ), 20-m linear sprint and change-of-direction (Pro-agility and V-cut tests) time, and aerobic capacity $\left(\dot{\mathrm{VO}}_{2 \mathrm{max}}\right.$, energy cost at LT, and velocity at $\dot{\mathrm{V}}_{2 \max }$ and $\mathrm{LT}$ ) on a level surface. Throughout the training sessions, HR during running did not differ between the groups (DRG: $77.7 \pm 4.6$ vs LRG: $76.4 \pm 4.6 \%$ of maximal HR; average across all sessions), while velocity was significantly higher for DRG $(14.5 \pm 1.1$ vs $12.0 \pm 1.9 \mathrm{~km} \cdot \mathrm{h}^{-1}$ ). After the training, DRG significantly improved knee extension torque at all angular velocities (9-24\%) and change-of-direction time for both tests (2-3\%), with no changes in other parameters. LRG significantly improved $\dot{\mathrm{V}} \mathrm{O}_{2 \max }(5 \%)$, energy cost at LT (3\%), and velocity at $\dot{\mathrm{VO}}_{2 \max }(7 \%)$, without changes in others. These results suggest that DR training has a greater potential to improve the knee extension strength and change-of-direction ability, but has little effect on the aerobic capacity, compared to HR-matched LR training.

Keywords: knee extension strength; change-of-direction ability; aerobic capacity; heart rate-matched running; eccentric component 


\section{Introduction}

In almost all types of sports, muscles contract both concentrically and eccentrically, where force is produced while a muscle-tendon complex is shortened and lengthened, respectively (Vogt and Hoppeler 2014). Concentric contractions provide positive work thereby moving the body toward an intended direction (Lindstedt et al. 2001), so concentric strength is indeed important for success in various sports (Cormie et al. 2011). Eccentric contractions provide negative work thereby absorbing mechanical energy (Lindstedt et al. 2001). Thus, eccentric strength is crucial when one needs to rapidly decelerate movements (Brughelli et al. 2008), where the absorbed energy is dissipated as heat (Lindstedt et al. 2001), or to perform a rapid change-of-direction (COD) or jump, where the absorbed energy is temporarily stored and then utilized in subsequent movements (Aagaard 2011). Indeed, the successive combination of eccentric and concentric contractions leads to better muscle performances than a concentric-only contraction, which is termed the stretch-shortening cycle (SSC) (Cormie et al. 2011; Vogt and Hoppeler 2014). Therefore, it is generally agreed that enhancing eccentric strength is highly beneficial for improving sports performances (Aagaard 2011; Vogt and Hoppeler 2014; Douglas et al. 2017).

Previous studies have shown that eccentric strength and/or jump performances are specifically improved by eccentric exercise training (Guilhem et al. 2010; Vogt and Hoppeler 2014; Douglas et al. 2017). Thus, various types of eccentric exercises have been increasingly implemented in athletic training programs (Guilhem et al. 2010; Vogt and Hoppeler 2014; Douglas et al. 2017). It is known that leg muscles, especially the knee extensors, predominantly perform eccentric contractions during downhill walking or downhill running (DR) to absorb shocks at each landing phase (Lindstedt et al. 2001). Several studies (Gault and Willems 2013; Rodio and Fattorini 2014; Maeo et al. 2016) have demonstrated that downhill, but not level or uphill (Rodio and Fattorini 2014), walking training can improve 
knee extension strength, including eccentric strength (Maeo et al. 2016). Cook et al. (2013) reported that DR training combined with other eccentric exercises was more effective than traditional resistance training in improving jump height; however, the training effect of DR itself is unclear since their intervention also included other eccentric exercises (Cook et al. 2013). Moreover, effects of DR training on other important sports performance-related parameters including, but not limited to, COD ability have never been examined. It is reported that the number of COD performed by a player during a single soccer match exceeded 700 times (Bloomfield et al. 2007), and that the COD ability was the most important factor for distinguishing between elite and sub-elite soccer players (Reilly et al. 2000). Considering the training effects of eccentric exercises (Guilhem et al. 2010; Vogt and Hoppeler 2014; Douglas et al. 2017) including downhill walking (Gault and Willems 2013; Rodio and Fattorini 2014; Maeo et al. 2016), together with the muscle action pattern during DR (i.e. rapid eccentric contractions), it is expected that DR training can improve knee extension strength and possibly jump and COD ability.

In addition to the abovementioned parameters, aerobic capacity is also of paramount importance for success in a wide range of sports (Helgerud et al. 2001). Not surprisingly, previous studies have well reported improvements in the aerobic capacity such as maximal oxygen uptake $\left(\dot{\mathrm{V}}_{2 \max }\right)$ and running economy at a given speed (e.g. the one associated with the lactate threshold [LT]) after endurance level running (LR) training (Garber et al. 2011). According to the American College of Sports Medicine position stand (Garber et al. 2011), the minimum training intensity range for improving the aerobic capacity is $64-76 \%$ of maximal heart rate $\left(\mathrm{HR}_{\max }\right)$ or $46-63 \%$ of $\dot{\mathrm{V}}_{2 \max }$ for untrained healthy individuals, whereas higher intensities are needed for trained athletes. For instance, HR associated with LT (HR $\mathrm{LT})$ is often used in endurance training (Mujika 2012), which is approximately $70-85 \% \mathrm{HR}_{\max }$ (Mujika 2012). Thus, it is reasonable to assume that the aerobic capacity can be improved by 
DR as well, if the training is performed at a certain intensity such as $\mathrm{HR}_{\mathrm{LT}}$. In addition, a large body of evidence exists (Balsalobre-Fernandez et al. 2016) supporting the positive effects of resistance or plyometric training on the aerobic capacity, at least partly owing to increased stiffness of muscle-tendon complex (i.e. better SSC utilization). Based on these, together with the potential training stimulus of DR on leg muscles as explained above, it is envisaged that DR training would also improve aerobic capacity similarly to, if not more effectively than, LR training.

The purpose of this study was therefore to examine the effects of DR versus LR training on various sports performance-related leg muscle performances including strength, power, jump height, and COD ability, as well as aerobic capacity. We hypothesized that DR and LR training at $\mathrm{HR}_{\mathrm{LT}}$ would result in greater improvements in the leg muscle performances for DR than LR training and similar improvements in the aerobic capacity. Since running is the most ubiquitous movement pattern in sports, understanding the effects of DR versus LR training may facilitate more effective training guidance and prescription.

\section{Materials and Methods}

\section{Participants}

This study was approved by the Ethics Committee of the National Institute of Fitness and Sports in Kanoya and was consistent with institutional ethical requirements for human experimentation in accordance with the Declaration of Helsinki. Prior to any assessments, the participants visited the laboratory and were fully informed about the procedures and possible risks involved as well as the purpose of the study, and written informed consent was obtained. A total of eighteen young males were recruited and allocated to either a DR (DRG: $n=10)$ or LR (LRG: $\mathrm{n}=8)$ group by matching $(P>0.05)$ the baseline mean $( \pm \mathrm{SD})$ age $(22.8 \pm 2.2$ years), height $(1.70 \pm 0.05 \mathrm{~m})$, and body mass $(65.4 \pm 5.6 \mathrm{~kg})$ between the groups to avoid 
any confounding factors. All participants were healthy and physically active, but none had been competitive athletes or involved in any type of systematic $\left(\geq 30 \mathrm{~min} \cdot \mathrm{day}^{-1}, \geq 2\right.$ days $^{\cdot}$ week $^{-1}$ ) resistance or aerobic training program in the past 12 months prior to the onset of the study. The participants were instructed to avoid any intensive and unfamiliar physical activities throughout the study period.

\section{Training program}

Training was conducted on a motorized treadmill (Quasar, h/p/cosmos, Germany) with either $-10 \%$ slope (DRG) or without slope (LRG) 3 sessions $\cdot w^{-1} e^{-1}$ for 5 weeks. The reason for choosing the $-10 \%$ slope for DRG is based on a recent review (Vernillo et al. 2017) that the energy cost of running is lowest (i.e. most economical) and plateaus between -10 and $-20 \%$, suggesting that this range is the optimal (representative) slope for DR training. Also, since a steeper slope may increase the risk of injuries including muscle damage (Maeo et al. 2017), we considered that $-10 \%$ was practically appropriate as a first step to investigate the DR training effects. Furthermore, to minimize potential detrimental effect of muscle damage, which is often induced by unaccustomed DR (Eston et al. 1995; Maeo et al. 2017), exercise duration at the initial three sessions was gradually increased from 5 to 10 and then 15 min. For the subsequent sessions, the training was conducted for $20 \mathrm{~min}$. Participants were instructed to report if they had any pain or soreness that may affect training, in which case they were asked to rest (avoid training). However, this never occurred and all participants completed all training sessions. During the training, HR was continuously recorded (RC3 GPS, Polar, Finland), and participants ran at their $\mathrm{HR}_{\mathrm{LT}}$. More specifically, the target HR was set for each participant as the HR at which LT occurred in the pre-training assessment (detailed below), and the running velocity was adjusted to achieve the target HR throughout the sessions. In our pilot study, 6 healthy young males $(26.2 \pm 2.5$ years $)$, who were 
comparable to the participants in DRG and LRG in terms of physical characteristics and $\dot{\mathrm{VO}}$ $2 \max \left(1.69 \pm 0.05 \mathrm{~m}, 67.8 \pm 6.4 \mathrm{~kg}, 52.8 \pm 3.6 \mathrm{ml} \cdot \mathrm{kg}^{-1} \cdot \mathrm{min}^{-1}\right.$, all $\left.P>0.05\right)$, performed incremental tests in both DR (-10\%) and LR conditions. The tests were conducted on separate days with a randomized order, during which $\mathrm{HR}$, blood lactate, and $\mathrm{V}_{2}$ were recorded while running velocity was gradually increased until exhaustion for LR (detailed below in the aerobic capacity section) and near-exhaustion for DR. The reason why the participants did not reach exhaustion in the DR condition was that we ended the test when they had reached near-exhaustion in order to avoid overspeed-induced accidents (e.g. tumbling) and injuries possibly induced by high mechanical stress during DR (Maeo et al. 2017). At LT for each condition, while velocity was significantly $(P=0.001)$ higher for DR $\left(14.9 \pm 0.6 \mathrm{~km} \cdot \mathrm{h}^{-1}\right)$ than LR $\left(11.7 \pm 1.0 \mathrm{~km} \cdot \mathrm{h}^{-1}\right)$, no significant differences were found in HR (DR vs LR: $79.6 \pm 4.4$ vs $\left.81.7 \pm 4.8 \% \mathrm{HR}_{\max }, P=0.362\right)$ and $\dot{\mathrm{VO}}{ }_{2}\left(69.0 \pm 9.5\right.$ vs $\left.73.0 \pm 7.8 \% \dot{\mathrm{VO}}_{2 \max }, P=0.139\right)$. Based on this, we assumed that the cardiovascular stress (i.e. $\dot{\mathrm{VO}}_{2}$ ) would be similar between DR and LR conditions at $\mathrm{HR}_{\mathrm{LT}}$. After each session, ratings of perceived exertion (RPE: Borg scale, 6-20 = very, very light - very, very hard) regarding each of the breathlessness and leg fatigue measures (Borg et al. 2010), as well as HR and running velocity, were recorded.

\section{Pre- and post-training assessments}

Before and after the interventions, participants were tested for various sports performance-related parameters, which were categorized into three as follows: 1) strength, power, and jump performance, 2) linear sprint and COD ability, and 3) aerobic capacity. These three categories were tested on different days, with at least 1 day apart, in each of the pre- and post-training assessments. The orders of the three categories were randomized, but were kept the same within each category. The pre- and post-training assessments were conducted within 7 days before and after the first and last training sessions, respectively. 
Each category consisted of the following parameters: 1) knee extension torque, leg extension power, and jump (squat, counter-movement, and rebound jumps) performance, 2) 20-m linear sprint, Pro-agility, and V-cut tests time, and 3) $\dot{\mathrm{V}} \mathrm{O}_{2 \max }$, energy cost at LT, and velocity at $\dot{\mathrm{VO}}_{2 \max }$ and LT. All assessments were conducted in the same indoor laboratory and gymnasium, and each assessment was conducted as follows.

\section{Strength, power, and jump performance}

Torque during knee extension with maximal eccentric, isometric, and concentric contractions was measured using an isokinetic dynamometer (Biodex system 2, Biodex Medical Systems, USA) (Maeo et al. 2016). Participants sat in an adjustable chair with support for the back and hips, with the hip joint kept at $\sim 80^{\circ}$ (anatomical position $=0^{\circ}$ ). The rotation axis of the knee was aligned with that of the dynamometer. The knee extension/flexion attachment on the dynamometer was set so that the range of motion of the knee joint was from 0 to $90^{\circ}$. Isometric torque was measured at $70^{\circ}$ of the knee joint. During the assessments, the torso, hips, and thighs were held tightly in the seat using adjustable lap belts to prevent extraneous movement. After sufficient warm-up and familiarization consisting of 3-5 submaximal to near-maximal contractions ( $\sim 50-90 \%$ effort) for each type of muscle actions, the participants performed maximal eccentric $\left(-150\right.$ and $\left.-30^{\circ} \cdot \mathrm{s}^{-1}\right)$, isometric $\left(0^{\circ} \cdot \mathrm{s}^{-1}\right)$, and concentric $(30$ and $150^{\circ} \cdot \mathrm{s}^{-1}$ ) contractions of knee extensors twice at each of the prescribed angular velocities, with the orders of contraction conditions and velocities randomized. Additional trials were performed if the difference in the peak torques of the two trials in each angular velocity was more than $10 \%$. A rest period of more than 3 min was taken between trials. In each angular velocity, the highest value of the peak torque was selected for analysis.

Maximal leg extension power was measured using an isokinetic leg extension dynamometer (Kick-Force, Takei, Japan) (Usui et al. 2016). Participants sat on the seat with 
support for the back, and the torso was held tightly in the seat. From the hip- and knee-flexed position both at $90^{\circ}$, participants performed leg extensions (i.e. simultaneous knee and hip extension) of both legs at a velocity of $0.8 \mathrm{~m} \cdot \mathrm{s}^{-1}$. After warm-up and practice of 3-5 trials with submaximal to near-maximal effort, trials with maximal effort were performed 5 times with an interval of at least $10 \mathrm{~s}$, and the highest value was adopted.

Squat, counter-movement, and rebound jump height was measured by using a mat switch platform system (Multi Jump Tester, DKH, Japan) (Usui et al. 2016). In this system, the jump height was calculated based on the participant's flight time. From a standing position on the mat $(66 \times 100 \mathrm{~cm})$, participants were instructed to perform jumps as high as possible and to land on the mat, while keeping their hands on both sides of the waist during the tasks. In the squat jump test, participants were in a squat position with the knee joints flexed at $90^{\circ}$, and jumped without a counter-movement. Trials with a detectable counter-movement were discarded. In the counter-movement jump test, participants jumped from a standing position using a counter-movement without restriction on joint angles. After practice of 3-5 trials with submaximal to near-maximal effort, the participants completed 2 maximal trials for each of these jump tests with a rest interval of at least $30 \mathrm{~s}$ between the trials. The highest height for the 2 trials for each test was used for analysis. In the rebound jump test, participants were instructed to jump as high as possible with minimum contact time 6 times in a row. After practice of 3-5 trials with submaximal to near-maximal effort, 2 maximal trials ( 6 jumps $\times 2$ trials) were conducted with at least 1 -min rest, and the rebound jump index was calculated as the ratio of jump height $\cdot$ contact time ${ }^{-1}$ for each jump. The highest index was adopted for analysis (Imai et al. 2016).

\section{Linear sprint and COD ability}

By using photoelectric cells (TC-System, Brower Timing Systems, USA) set at $0.4 \mathrm{~m}$ from 
the floor, 20-m linear sprint time was measured ( $\pm 1 / 1000 \mathrm{~s})$. After warm-up of submaximal running and stretching followed by $2-3$ practice trials with near-maximal effort, 2 trials with maximal effort were performed with at least 1-min rest, and the shortest sprint time was adopted for analysis.

Following the linear sprint test, Pro-agility and V-cut test time were measured using the same photoelectric cells. Schematic illustrations of these tests are shown in Figure 1. In the Pro-agility test, each participant straddled the middle (start) line and sprinted in the right direction for $5 \mathrm{~m}$, changed direction and sprinted back for $10 \mathrm{~m}$ to another line and pivoted back and sprinted through the start line for $5 \mathrm{~m}$ (Magal et al. 2009). In the V-cut test, the participants performed a $25-\mathrm{m}$ sprint with $45^{\circ} \mathrm{COD}$ every $5 \mathrm{~m}$ by stepping between each pair of cones separated by $0.7 \mathrm{~m}$ (Tous-Fajardo et al. 2016). For both tests, participants were instructed to start from the same position between trials, run as fast as possible, and touch or pass the line with the foot at each turn. After 2-3 practice trials with near-maximal effort, 2 trials with maximal effort were performed with at least 3-min rest, and the shortest time was adopted for analysis. Additional trials were performed if considered invalid (e.g. when participants slipped or did not touch or pass the lines).

\section{Aerobic capacity}

$\dot{\mathrm{V}} \mathrm{O}_{2 \max }$, energy cost at LT, and velocity at $\dot{\mathrm{VO}}_{2 \max }$ and LT were assessed by multi-stage and incremental tests performed on the same treadmill as used in the training set at $0 \%$ slope. The multi-stage test consisted of running for $4 \mathrm{~min}$ at each of several stages (explained below), with a rest period of 1 min between stages. Running velocity at the first stage was set at $7.2-12 \mathrm{~km} \cdot \mathrm{h}^{-1}$ depending on each participant's physical fitness level, and it was increased by $1.2 \mathrm{~km} \cdot \mathrm{h}^{-1}\left(20 \mathrm{~m} \cdot \mathrm{min}^{-1}\right)$ every stage (every $\left.4 \mathrm{~min}\right)$ until blood lactate concentration exceeded $4.0 \mathrm{mmol} \cdot \mathrm{L}^{-1}$ [adapted from (Helgerud et al. 2001; Storen et al. 2008; Folland et al. 2017)]. 
Before the multi-stage test, the participants conducted warm-up consisting of stretching and jogging for $10 \mathrm{~min}$ in total, and they completed 5-7 stages in the test. Following the multi-stage test and a rest period of $10 \mathrm{~min}$, the incremental test was conducted. The starting velocity in the incremental test was the same as the one used in the second last stage in the multi-stage test for each participant. The velocity was subsequently increased by $0.6 \mathrm{~km} \cdot \mathrm{h}^{-1}$ $\left(10 \mathrm{~m} \cdot \mathrm{min}^{-1}\right)$ every $1 \mathrm{~min}$ until volitional exhaustion [adapted from (Folland et al. 2017; Sumi et al. 2017)], which was defined as the point when the participant could no longer run at the required velocity. HR was continuously recorded (RC3 GPS, Polar, Finland) during both tests.

$\dot{\mathrm{V}} \mathrm{O}_{2}$ was measured using the Douglas bag method with a dry-gas meter (NDS-2A-T, Shinagawa CORP, Japan) and gas analyzer (VmaxS 29C, Sensor-medics, USA), which was calibrated before each test using reference gases of known concentrations. Expired gas was collected at the last $1 \mathrm{~min}$ of each stage in the multi-stage test and every $1 \mathrm{~min}$ or every $30 \mathrm{~s}$ (when reaching exhaustion) for the last 3-4 min in the incremental test. Criteria for attainment of $\dot{\mathrm{VO}}_{2 \max }$ included at least two of the following: 1) a leveling off in $\dot{\mathrm{VO}}_{2}$ despite an increase in a treadmill velocity, 2) maximal respiratory exchange ratio $\geq 1.1,3$ ) $\mathrm{HR} \geq 90 \%$ of the age predicted $\mathrm{HR}_{\max }\left(220\right.$ - age), and 4) blood lactate $\geq 8 \mathrm{mmol} \cdot \mathrm{L}^{-1}$ (Howley et al. 1995). Blood sample was collected (Lactate Pro2 LT -1730, Arkray, Japan) immediately after each stage in the multi-stage test and 1 and 3 min after the completion of the incremental test. LT was calculated using software (MEQNET LT Manager, Arkray, Japan), defined as the intersection of two linear lines in plot of lactate versus running velocity where the residual sum of squares for both lines added together was minimized. The energy cost was calculated from respiratory exchange ratio data and updated non-protein respiratory quotient equations (Peronnet and Massicotte 1991). The energy derived from each substrate was calculated by multiplying fat and carbohydrate usage by 9.75 and $4.07 \mathrm{kcal}$, respectively. 


\section{Statistical analysis}

Descriptive data are presented as means \pm SDs. All data were analyzed using SPSS software (version 23.0, IBM Corp, USA). The normality of the data was checked and subsequently confirmed by a Shapiro-Wilk's test for all variables except for RPE. Thus, non-parametric tests were used for RPE comparisons. HR and running velocity throughout the training sessions were compared by a two-way ( 2 groups $\times 15$ sessions $)$ repeated measures analysis of variance (ANOVA). When a significant interaction effect was found, an unpaired t-test was used to test the difference between the groups for each session. Comparisons between sessions were not made since it was not the purpose of this study. RPE for breathlessness and leg fatigue measures were compared between the groups at each session by a Mann-Whitney $\mathrm{U}$ Test. For the variables measured before and after the interventions, an unpaired t-test was used to test the differences between the groups in the initial values for each variable. Changes in knee extension torque before and after the interventions were compared by a two-way ( 2 times $\times 5$ angular velocities) ANOVA for each group. Changes in other variables before and after the interventions were compared by a paired t-test for each group. Statistical significance was set at $P<0.05$. As indices of effect size, Cohen's $d$ values were calculated and presented where significant, and interpreted as large: $\geq 0.80$, medium: $0.50-0.79$, small: 0.20-0.49, and trivial: $<0.20$ (Faul et al. 2007).

\section{Results}

\section{$H R$, running velocity, and RPE during training}

No significant main effect of group $(P=0.468)$ or interaction $(P=0.229)$ was found in HR (Figure 2a). The average HRs across all sessions were $77.7 \pm 4.6$ for DRG and $76.4 \pm$ $4.6 \% \mathrm{HR}_{\max }$ for $\mathrm{LRG}$. 
A significant interaction $(P<0.001)$ was found in the running velocity (Figure $2 b$ ), which was always higher for DRG than LRG at all sessions $(14.5 \pm 1.1 \mathrm{vs} 12.0 \pm 1.9 \mathrm{~km} / \mathrm{h}$; average across all sessions, $d=1.56)$.

RPE for breathlessness measures was not different between the groups at all sessions except for the 4th, which was higher for DRG than LRG $(P=0.018, d=1.17$, Figure $2 \mathrm{c})$. RPE for leg fatigue measures was significantly higher for DRG than LRG at the sessions from 2 nd to 8 th $(P=0.002-0.015, d=1.53-2.17$, Figure $2 \mathrm{~d})$.

\section{Pre- and post-training assessments}

No significant baseline differences were found between the groups in any of the variables $(P$ $\geq 0.21$ ). All, except for one, participants completed all assessments. One participant in DRG could not complete the post-training aerobic capacity test due to pain in the ankle joint which occurred during the post-test. Consequently, the number of the participants in DRG in the following section is 9 for the aerobic capacity-related variables and 10 for the others.

Strength, power, and jump performance

Knee extension torque for DRG had significant main effects of time $(P=0.007)$ and angular velocity $(P<0.001)$ without their interaction $(P=0.201$, Figure 3$)$, indicating that the knee extension torque significantly increased at all angular velocities $(9-24 \%, d=0.33-1.00)$. No significant main effect or interaction was found in the knee extension torque for LRG $(P>$ $0.05)$.

No significant changes were found in the leg extension power in both DRG (pre vs post: $1262 \pm 117$ vs $1305 \pm 114 \mathrm{~W}, P=0.103)$ and $L R G(1151 \pm 238$ vs $1178 \pm 228 \mathrm{~W}, P=$ $0.339)$.

No significant changes were found in any of the squat jump height (DRG: $35.3 \pm 4.9$ vs 
$36.0 \pm 6.0 \mathrm{~cm}, P=0.451, \mathrm{LRG}: 36.7 \pm 3.9$ vs $36.4 \pm 4.3 \mathrm{~cm}, P=0.804)$, counter-movement jump height (DRG: $40.1 \pm 6.0$ vs $40.8 \pm 7.4 \mathrm{~cm}, P=0.455$, LRG: $40.6 \pm 3.6$ vs $40.3 \pm 3.9 \mathrm{~cm}$, $P=0.665$ ), and rebound jump index (DRG: $1.95 \pm 0.47$ vs $1.81 \pm 0.35 \mathrm{~cm} \cdot \mathrm{s}^{-1}, P=0.112$ LRG: $2.25 \pm 0.53$ vs $2.06 \pm 0.49 \mathrm{~cm} \cdot \mathrm{s}^{-1}, P=0.052$ ) for both groups.

\section{Linear sprint and COD ability}

No significant changes were found in the 20-m linear sprint time in DRG (pre vs post: $3.13 \pm$ 0.16 vs $3.12 \pm 0.14 \mathrm{~s}, P=0.696)$ and LRG $(3.12 \pm 0.05$ vs $3.13 \pm 0.09 \mathrm{~s}, P=0.717)$.

Significant improvements were found in DRG in both of the Pro-agility $(3 \%, P=$ $0.007, d=0.67)$ and V-cut $(2 \%, P=0.036, d=0.31)$ test time (Figure $4 \mathrm{a}$ and $\mathrm{b})$. No such changes were found in LRG $(P=0.213-0.403)$.

\section{Aerobic capacity}

No significant changes were found in any of the variables in DRG $(P>0.05$, Figure 5 and 6$)$. LRG significantly improved $\dot{\mathrm{VO}}_{2 \max }\left(5 \%, P=0.008, d=0.42\right.$, Figure 5a), velocity at $\dot{\mathrm{VO}}_{2 \max }$ $(7 \%, P=0.001, d=0.65$, Figure $5 \mathrm{~b})$, and energy cost at LT $(3 \%, P=0.003, d=0.72$, Figure 6a), but not velocity at LT $(P=0.080$, Figure $6 \mathrm{~b})$.

\section{Discussion}

The main findings obtained here were that after the HR-matched DR and LR training, 1) only DR training improved knee extension strength and COD ability, and 2) only LR training improved aerobic capacity. These partly supported our hypotheses. Collectively, the results suggest that DR training has a greater potential to improve knee extension strength and COD ability, but its effect on the aerobic capacity is little, compared to HR-matched LR training.

Throughout all training sessions, HR during running did not differ between the groups 
(Figure 2a). This is not surprising since we intentionally matched it and manipulated the running velocity according to the target $\mathrm{HR}$ values. As a consequence, the running velocity was always higher for DRG than LRG (Figure 2b), which well agrees with that reviewed somewhere else (Vernillo et al. 2017). Aligning with these, at least in part, RPE for breathlessness was similar between the groups at all sessions except for the 4th (Figure 2c), and RPE for leg fatigue measures was greater at several (from the 2nd to 8th) sessions for DRG than LRG (Figure 2d). Taken together with a similar $\dot{\mathrm{VO}} 2$ between the DR and LR conditions as observed in our pilot study (discussed later), these results suggest that the training stimuli on the cardiovascular system were similar between the groups and those to the leg muscles were greater for DRG than LRG.

Knee extension torque in all contraction conditions significantly improved in DRG only (Figure 3). This is in line with previous studies that showed significant improvements in knee extension strength after downhill (Gault and Willems 2013; Rodio and Fattorini 2014; Maeo et al. 2015; Maeo et al. 2016), but not after level (Rodio and Fattorini 2014), walking training. Using data available from a review article (Guilhem et al. 2010), we calculated that strength gains per session following isotonic eccentric training of the knee extensors in untrained individuals were $2.8 \pm 1.4 \%$ (mean $\pm \mathrm{SD}$ for several studies) for eccentric, $0.8 \pm$ $0.5 \%$ for isometric, and $1.3 \pm 0.9 \%$ for concentric strength. The corresponding values in this study were $1.4 \%$ (average of -150 and $-30^{\circ} \cdot \mathrm{s}^{-1}$ ), $0.6 \%$, and $1.4 \%$ (average of 150 and $30^{\circ} \cdot \mathrm{s}^{-1}$ ), which are within the range (SD) of those of the previous studies. These results indicate that DR training can improve knee extension strength in all contraction conditions as effectively as other forms of eccentric training can. Furthermore, the COD ability, as assessed by the Pro-agility and V-cut tests, also significantly improved in DRG only (Figure 4). The current results support the usefulness of DR training for simultaneously improving both knee extension strength and COD ability. These improvements would be mainly attributable to 
considerably high repetitions of braking muscle actions in the leg muscles, especially the knee extensors (Vernillo et al. 2017), performed during DR training, although we do not have any data explaining possible mechanisms. Despite a wealth of research exploring neural, reflex, and/or muscle-tendon adaptations following traditional high-intensity resistance or eccentric training (Aagaard 2011; Vogt and Hoppeler 2014; Douglas et al. 2017), mechanisms underpinning performance improvements induced by endurance-type eccentric training such DR are yet to be clarified. It is worth exploring whether DR training induces similar adaptations to resistance training, such as increased neural drive and/or muscle size. Future studies should be directed toward the identification of the neuromuscular adaptations following DR training.

On the other hand, no changes were observed in other variables including jump performances in DRG. This somewhat contradicts with our hypothesis and previous reports (Vogt and Hoppeler 2014; Douglas et al. 2017) that eccentric training is effective in improving jump performances. It should be borne in mind that a training-induced performance improvement tends to be most clearly observed in tasks similar to the training task, and this is called "the training- or task-specificity" (Sale 1988; Usui et al. 2016). Given that DR and COD tasks both involve "running" and "braking muscle actions", it is likely that the training effect conferred by DR was transferred to the COD ability, but not to the jump and other performances, owing to the similarity between the tasks. At the same time, this highlights that a training task should closely mimic the demands of the athletic tasks depending on a primary training goal. Without considering such aspect, incorporating an eccentric exercise itself in a training program could be meaningless. These findings may have application for optimizing training prescription for a variety of sports based on a specific training goal.

The aerobic capacity significantly improved in LRG only. This improvement in LRG is 
reasonable considering that the training intensity in this study $\left(\sim 77 \% \mathrm{HR}_{\max }\right)$ was above the suggested minimum intensity for untrained individuals $\left(64-76 \% \mathrm{HR}_{\max }\right)($ Garber et al. 2011). On the other hand, it is unknown why DRG could not improve aerobic capacity. We observed an equivalent $\dot{\mathrm{VO}}_{2}$ at $\mathrm{HR}_{\mathrm{LT}}$ between the DR and LR conditions in our pilot study, as well as similar RPE for breathlessness measures throughout the training sessions (Figure 2c), suggesting equivalent training stimuli on the cardiovascular system between both groups in this study. While difficult to explain, a recent study (Schlagowski et al. 2016) showed that mitochondrial function in rat leg muscles was improved by uphill running (UR) training, but not by DR training, performed at the same $\mathrm{V}_{2}$, at least partly aligning with our result. As the previous study suggested (Schlagowski et al. 2016), it is possible that muscle damage induced by DR may have delayed and/or offset the mitochondrial adaptations to the training. However, it seems unlikely to be the main reason since we gradually increased the exercise duration in the initial training sessions to minimize such damage. Indeed, although a few participants reported mild muscle soreness after the first session, no participant complained about soreness thereafter throughout the intervention. Nevertheless, assessments of objective muscle damage indices (e.g. plasma creatine kinase activities) at least at pre- and post-tests would provide more concrete evidence regarding this issue, thus should be included in future studies.

Another possibility for the lack of improvement in the aerobic capacity of DRG may be due to biomechanical differences between DR and LR. It is known that joint kinematics and kinetics, as well as SSC utilization, differ between DR and LR (Vernillo et al. 2017), and a recent study (Folland et al. 2017) reported that running technique is an important component of the aerobic capacity. Since the aerobic capacity test was conducted under the LR condition in this study, it is possible that LRG, but not DRG, may have adapted to run with more efficient movement patterns and/or SSC utilization under the LR condition, which can be 
considered as the "slope-specificity". In this sense, it is interesting to explore if DR training would improve the aerobic capacity tested under the DR condition to a greater extent, if any, than LR would, although challenging given the possible risks of injuries. Alternatively, testing the aerobic capacity under a condition unrelated to either LR or DR, for instance by using a cycling ergometer, may shed light on the effect of LR and DR by excluding the potential slope-specificity effect. More research is needed to clarify the effects of DR on the aerobic capacity with various types of approaches.

We did not set an UR training group, so its effects on the variables measured in this study are unknown. However, it is reported that UR or LR training both resulted in 1) no changes in the knee extension strength (Ferley et al. 2014), but 2) significant improvements in the aerobic capacity tested under the level condition, with a greater degree after the LR than UR training (Ferley et al. 2013). These and present findings collaboratively suggest that muscle strength and related performances (e.g. COD ability) can be specifically improved by DR training, whereas the aerobic capacity during LR can be most effectively improved by LR training. To substantiate these issues, it is necessary to directly compare the training effect of UR, DR, and LR using the same test setting. Furthermore, in a practical point of view, it is of great importance to explore whether a combination of these training can simultaneously improve muscular and aerobic performances. Further research is needed to clarify these.

In conclusion, this study for the first time revealed that heart rate-matched downhill versus level running training resulted in distinct performance improvements. Downhill, but not level, running training was effective in improving knee extension strength and change-of-direction ability, while both failed to enhance leg extension power, jump performance, and linear sprint time. On the other hand, only level running training improved the aerobic capacity. These results suggest that downhill running training has a greater potential to improve knee extension strength and change-of-direction ability, but its effect on 
the aerobic capacity is little, compared to heart rate-matched level running training.

\section{Acknowledgement}

This work was supported by a Grant-in-Aid for Japan Society for the Promotion of Science Research Fellow (15J03228).

\section{Conflict of interest}

The authors declare no conflict of interest, and that no companies or manufacturers will benefit from the results of this study. 


\section{REFERENCES}

Aagaard, P. 2011. Neural adaptations to resistance exercise. In Strength and conditioning: biological principles and practical applications. Edited by M. Cardinale, R. Newton, and K. Nosaka. John Wiley \& Sons. Chichester, West Sussex, UK. pp. 105-124.

Balsalobre-Fernandez, C., Santos-Concejero, J., and Grivas, G.V. 2016. Effects of strength training on running economy in highly trained runners: A systematic review with meta-analysis of controlled trials. J. Strength Cond. Res. 30: 2361-8. doi:10.1519/JSC.0000000000001316.

Bloomfield, J., Polman, R., and O'Donoghue, P. 2007. Physical demands of different positions in FA Premier League Soccer. J. Sports Sci. Med. 6: 63-70.

Borg, E., Borg, G., Larsson, K., Letzter, M., and Sundblad, B.M. 2010. An index for breathlessness and leg fatigue. Scand. J. Med. Sci. Sports, 20: 644-50. doi:10.1111/j.1600-0838.2009.00985.x.

Brughelli, M., Cronin, J., Levin, G., and Chaouachi, A. 2008. Understanding change of direction ability in sport: a review of resistance training studies. Sports Med. 38: 1045-63. doi:10.2165/00007256-200838120-00007.

Cook, C.J., Beaven, C.M., and Kilduff, L.P. 2013. Three weeks of eccentric training combined with overspeed exercises enhances power and running speed performance gains in trained athletes. J. Strength Cond. Res. 27: 1280-6. doi:10.1519/JSC.0b013e3182679278.

Cormie, P., McGuigan, M.R., and Newton, R.U. 2011. Developing maximal neuromuscular power: Part 1--biological basis of maximal power production. Sports Med. 41: 17-38. doi:10.2165/11537690-000000000-00000.

Douglas, J., Pearson, S., Ross, A., and McGuigan, M. 2017. Chronic adaptations to eccentric training: A systematic review. Sports Med. 47: 917-941. doi:10.1007/s40279-016-0628-4.

Eston, R.G., Mickleborough, J., and Baltzopoulos, V. 1995. Eccentric activation and muscle damage: biomechanical and physiological considerations during downhill running. Br. J. Sports Med. 29: 89-94.

Faul, F., Erdfelder, E., Lang, A.G., and Buchner, A. 2007. G*Power 3: a flexible statistical power analysis program for the social, behavioral, and biomedical sciences. Behav. Res. Methods, 39: 175-91. 
Ferley, D.D., Osborn, R.W., and Vukovich, M.D. 2013. The effects of uphill vs. level-grade high-intensity interval training on VO2max, Vmax, V(LT), and Tmax in well-trained distance runners. J. Strength Cond. Res. 27: 1549-59. doi:10.1519/JSC.0b013e3182736923.

Ferley, D.D., Osborn, R.W., and Vukovich, M.D. 2014. The effects of incline and level-grade high-intensity interval treadmill training on running economy and muscle power in well-trained distance runners. J. Strength Cond. Res. 28: 1298-309. doi:10.1519/JSC.0000000000000274.

Folland, J.P., Allen, S.J., Black, M.I., Handsaker, J.C., and Forrester, S.E. 2017. Running Technique is an Important Component of Running Economy and Performance. Med. Sci. Sports Exerc. 49: 1412-1423. doi:10.1249/MSS.0000000000001245.

Garber, C.E., Blissmer, B., Deschenes, M.R., Franklin, B.A., Lamonte, M.J., Lee, I.M., et al. 2011. American College of Sports Medicine position stand. Quantity and quality of exercise for developing and maintaining cardiorespiratory, musculoskeletal, and neuromotor fitness in apparently healthy adults: guidance for prescribing exercise. Med. Sci. Sports Exerc. 43: 1334-59. doi:10.1249/MSS.0b013e318213fefb.

Gault, M.L. and Willems, M.E. 2013. Isometric strength and steadiness adaptations of the knee extensor muscles to level and downhill treadmill walking in older adults. Biogerontology, 14: 197-208. doi:10.1007/s10522-013-9423-x.

Guilhem, G., Cornu, C., and Guevel, A. 2010. Neuromuscular and muscle-tendon system adaptations to isotonic and isokinetic eccentric exercise. Ann. Phys. Rehabil. Med. 53: 319-41. doi:10.1016/j.rehab.2010.04.003.

Helgerud, J., Engen, L.C., Wisloff, U., and Hoff, J. 2001. Aerobic endurance training improves soccer performance. Med. Sci. Sports Exerc. 33: 1925-31.

Howley, E.T., Bassett, D.R., Jr., and Welch, H.G. 1995. Criteria for maximal oxygen uptake: review and commentary. Med. Sci. Sports Exerc. 27: 1292-301.

Imai, A., Kaneoka, K., Okubo, Y., and Shiraki, H. 2016. Immediate effects of different trunk exercise programs on jump performance. Int. J. Sports Med. 37: 197-201. doi: $10.1055 / \mathrm{s}-0035-1559714$.

Lindstedt, S.L., LaStayo, P.C., and Reich, T.E. 2001. When active muscles lengthen: properties and consequences of eccentric contractions. News Physiol. Sci. 16: 256-61.

Maeo, S., Ando, Y., Kanehisa, H., and Kawakami, Y. 2017. Localization of damage in the human leg muscles induced by downhill running. Sci. Rep. 7: 5769. 
doi:10.1038/s41598-017-06129-8.

Maeo, S., Yamamoto, M., and Kanehisa, H. 2015. Muscular adaptations to short-term low-frequency downhill walking training. Int. J. Sports Med. 36: 150-6. doi:10.1055/s-0034-1385885.

Maeo, S., Yamamoto, M., and Kanehisa, H. 2016. Downhill walking training with and without exercise-induced muscle damage similarly increase knee extensor strength. J. Sports Sci. 34: 2018-26. doi:10.1080/02640414.2016.1149607.

Magal, M., Smith, R.T., Dyer, J.J., and Hoffman, J.R. 2009. Seasonal variation in physical performance-related variables in male NCAA Division III soccer players. J. Strength Cond. Res. 23: 2555-9. doi:10.1519/JSC.0b013e3181b3ddbf.

Mujika, I. 2012. Endurance training: science and practice. Inigo Mujika, Vitoria-Gasteiz.

Peronnet, F. and Massicotte, D. 1991. Table of nonprotein respiratory quotient: an update. Can J. Sport Sci. 16: 23-9.

Reilly, T., Williams, A.M., Nevill, A., and Franks, A. 2000. A multidisciplinary approach to talent identification in soccer. J. Sports Sci. 18: 695-702. doi:10.1080/02640410050120078.

Rodio, A. and Fattorini, L. 2014. Downhill walking to improve lower limb strength in healthy young adults. Eur. J. Sport Sci. 14: 806-12. doi:10.1080/17461391.2014.908958.

Sale, D.G. 1988. Neural adaptation to resistance training. Med. Sci. Sports Exerc. 20: S135-45.

Schlagowski, A.I., Isner-Horobeti, M.E., Dufour, S.P., Rasseneur, L., Enache, I., Lonsdorfer-Wolf, E., et al. 2016. Mitochondrial function following downhill and/or uphill exercise training in rats. Muscle Nerve, 54: 925-935. doi:10.1002/mus.25144.

Storen, O., Helgerud, J., Stoa, E.M., and Hoff, J. 2008. Maximal strength training improves running economy in distance runners. Med. Sci. Sports Exerc. 40: 1087-92. doi:10.1249/MSS.0b013e318168da2f.

Sumi, D., Kojima, C., and Goto, K. 2017. Impact of Endurance Exercise in Hypoxia on Muscle Damage, Inflammatory and Performance Responses. J. Strength Cond. Res. Published Ahead of Print. doi:10.1519/JSC.0000000000001911.

Tous-Fajardo, J., Gonzalo-Skok, O., Arjol-Serrano, J.L., and Tesch, P. 2016. Enhancing change-of-direction speed in soccer players by functional inertial eccentric overload and 
vibration training. Int. J. Sports Physiol. Perform. 11: 66-73. doi:10.1123/ijspp.2015-0010.

Usui, S., Maeo, S., Tayashiki, K., Nakatani, M., and Kanehisa, H. 2016. Low-load slow movement squat training increases muscle size and strength but not power. Int. J. Sports Med. 37: 305-12. doi:10.1055/s-0035-1564255.

Vernillo, G., Giandolini, M., Edwards, W.B., Morin, J.B., Samozino, P., Horvais, N., et al. 2017. Biomechanics and physiology of uphill and downhill running. Sports Med. 47: 615-629. doi:10.1007/s40279-016-0605-y.

Vogt, M. and Hoppeler, H.H. 2014. Eccentric exercise: mechanisms and effects when used as training regime or training adjunct. J. Appl. Physiol. 116: 1446-1454. doi:10.1152/japplphysiol.00146.2013. 


\section{FIGURE LEGENDS}

Figure 1. Schematic illustrations of the Pro-agility (left) and V-cut tests (right).

Figure 2. Heart rate (a), running velocity (b), and ratings of perceived exertion (RPE) for breathlessness (c) and leg fatigue measures (d) throughout the training sessions. DRG: downhill running group (closed circle), LRG: level running group (open circle). Values are means \pm SDs. *A significant difference between groups at $P<0.05$.

Figure 3. Maximal knee extension torque before (pre: circle) and after (post: square) the interventions. DRG: downhill running group, LRG: level running group. Values are means \pm SDs. *A significant increase from pre at $P<0.05$.

Figure 4. Pro-agility (a) and V-cut (b) test time before (pre) and after (post) the interventions. DRG: downhill running group (closed bars), LRG: level running group (open bars). Values are means \pm SDs. $*$ A significant decrease from pre at $P<0.05$

Figure 5. Maximal oxygen uptake $\left(\dot{\mathrm{V}}_{2 \max }\right.$ : a) and velocity at $\dot{\mathrm{V}}_{2 \max }$ (b) before (pre) and after (post) the interventions. DRG: downhill running group (closed bars), LRG: level running group (open bars). Values are means \pm SDs. *A significant change from pre at $P<$ 0.05 .

Figure 6. Energy cost at lactate threshold (LT) (a) and velocity at LT (b) before (pre) and after (post) the interventions. DRG: downhill running group (closed bars), LRG: level running group (open bars). Values are means \pm SDs. *A significant change from pre at $P<$ 0.05 . 


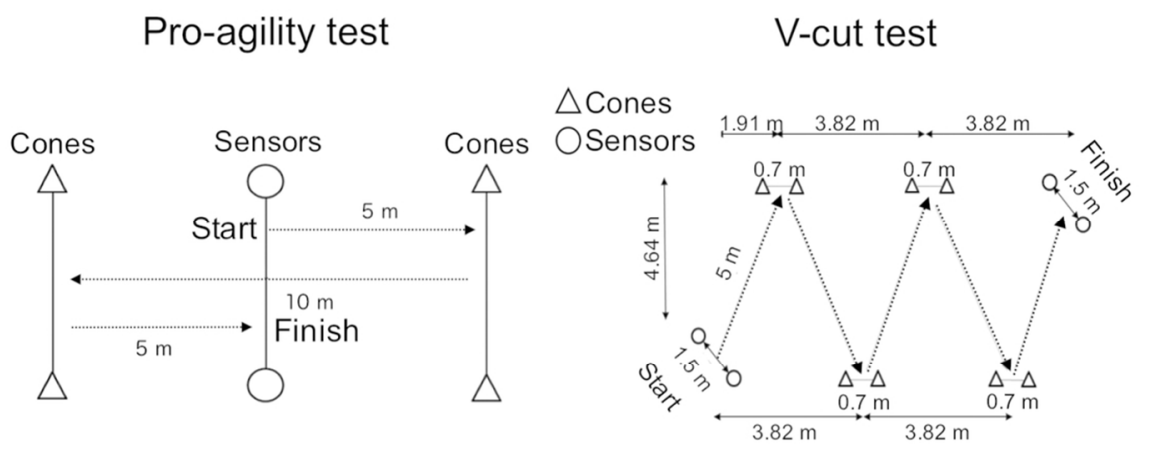

Figure 1. Schematic illustrations of the Pro-agility (left) and V-cut tests (right). $95 \times 126 \mathrm{~mm}(300 \times 300 \mathrm{DPI})$ 
a

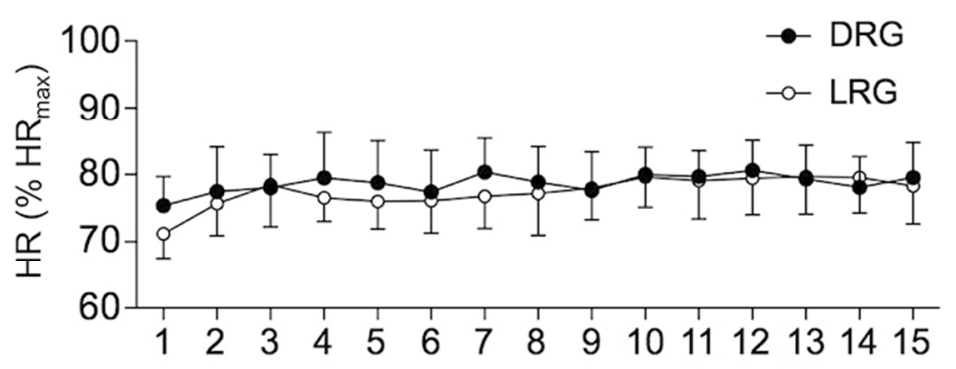

b

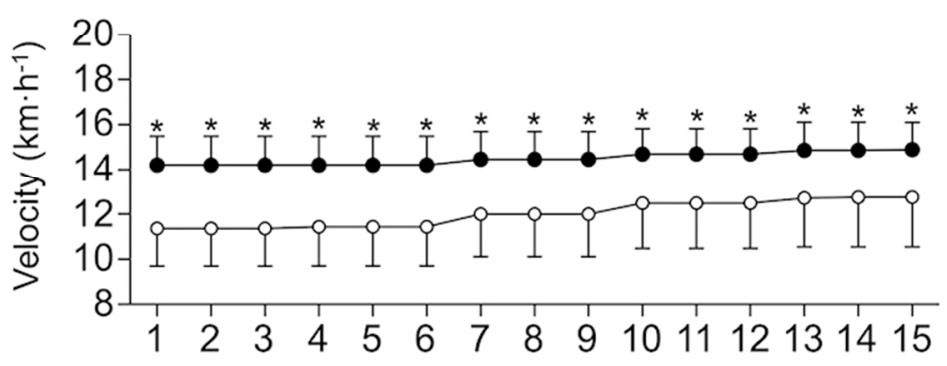

C

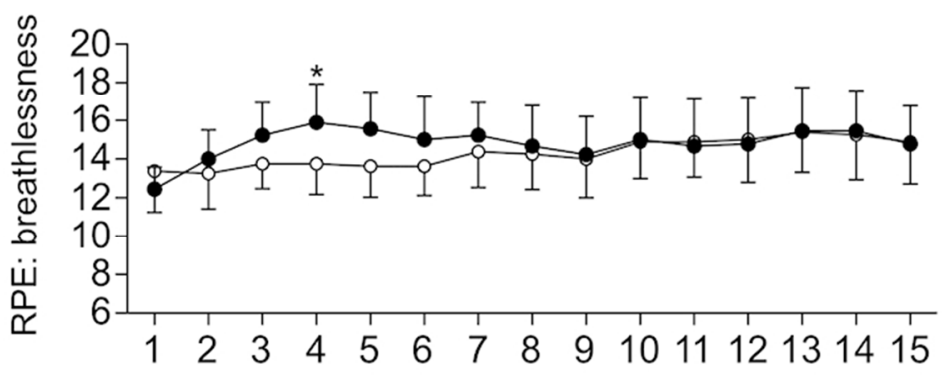

d

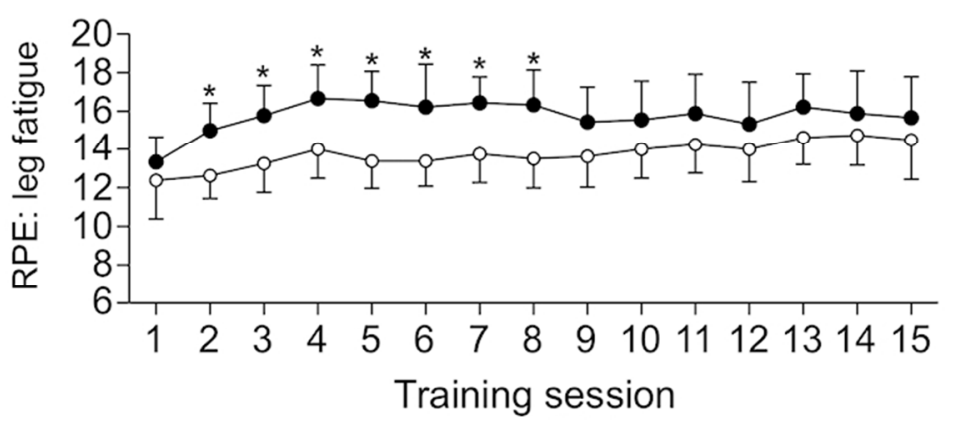

Figure 2. Heart rate (a), running velocity (b), and ratings of perceived exertion (RPE) for breathlessness (c) and leg fatigue (d) throughout the training sessions. DRG: downhill running group (closed circle), LRG: level running group (open circle). Values are means \pm SDs. ${ }^{*} A$ significant difference between groups at $P<0.05$.

$75 \times 99 \mathrm{~mm}(300 \times 300 \mathrm{DPI})$ 

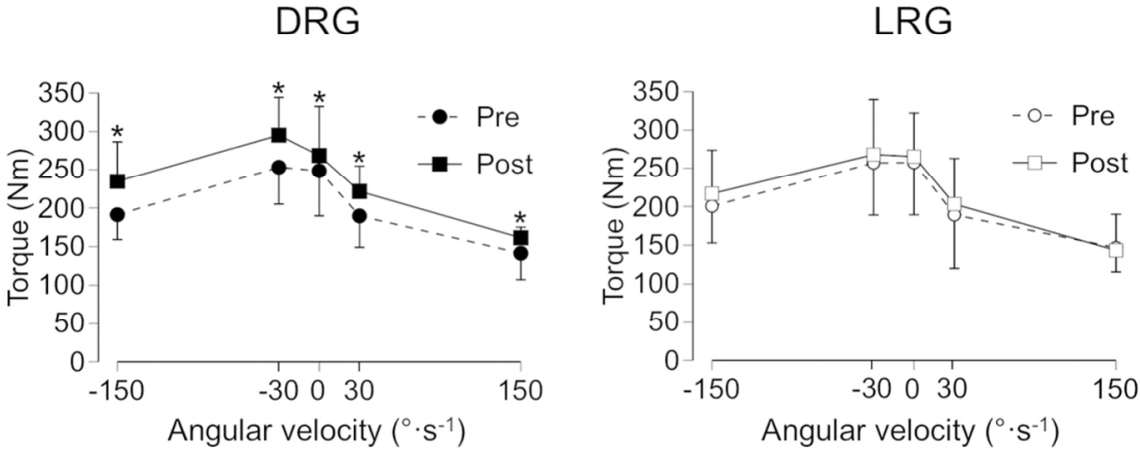

Figure 3. Maximal knee extension torque before (pre: circle) and after (post: square) the interventions. DRG: downhill running group, LRG: level running group. Values are means \pm SDs. *A significant increase from pre at $\mathrm{P}<0.05$.

$95 \times 126 \mathrm{~mm}(300 \times 300 \mathrm{DPI})$ 
a

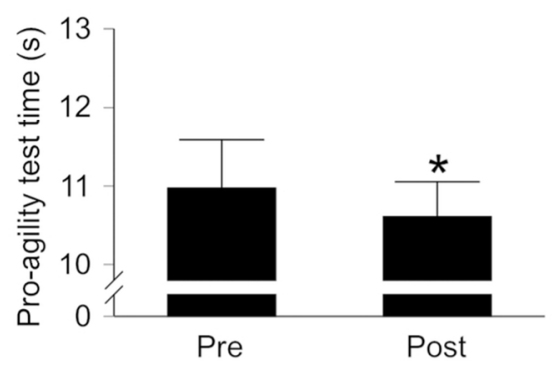

b

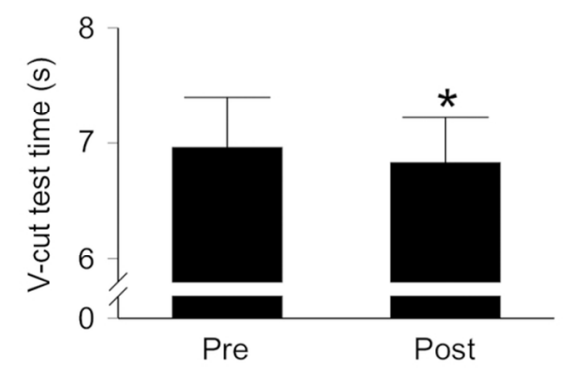

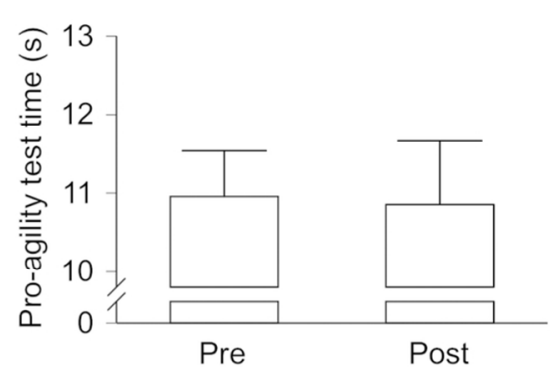

LRG

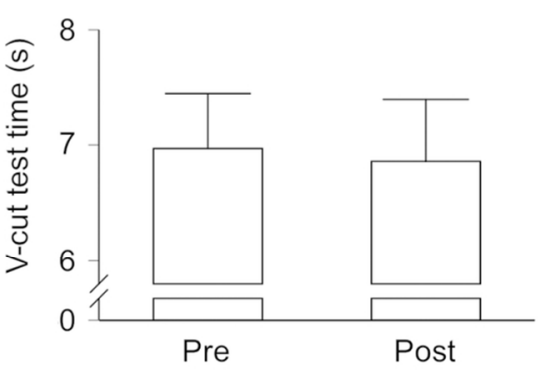

Figure 4. Pro-agility (a) and V-cut (b) test time before (pre) and after (post) the interventions. DRG: downhill running group (closed bars), LRG: level running group (open bars). Values are means \pm SDs. $* A$ significant decrease from pre at $\mathrm{P}<0.05$.

$95 \times 126 \mathrm{~mm}(300 \times 300 \mathrm{DPI})$ 

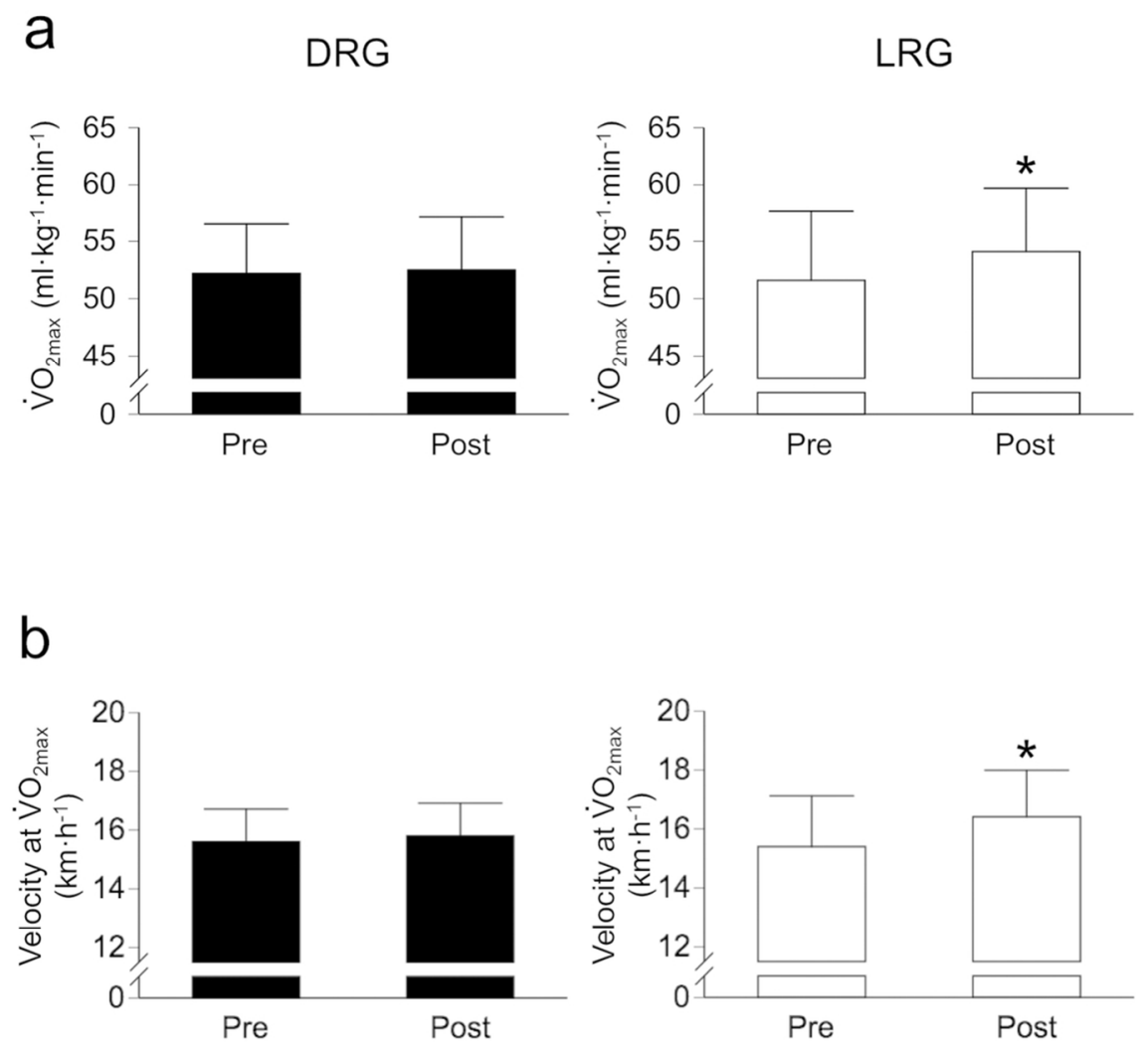

Figure 5. Maximal oxygen uptake (VO2max: a) and velocity at VO2max (b) before (pre) and after (post) the interventions. DRG: downhill running group (closed bars), LRG: level running group (open bars). Values are means \pm SDs. ${ }^{*} A$ significant change from pre at $P<0.05$.

$95 \times 126 \mathrm{~mm}(300 \times 300 \mathrm{DPI})$ 

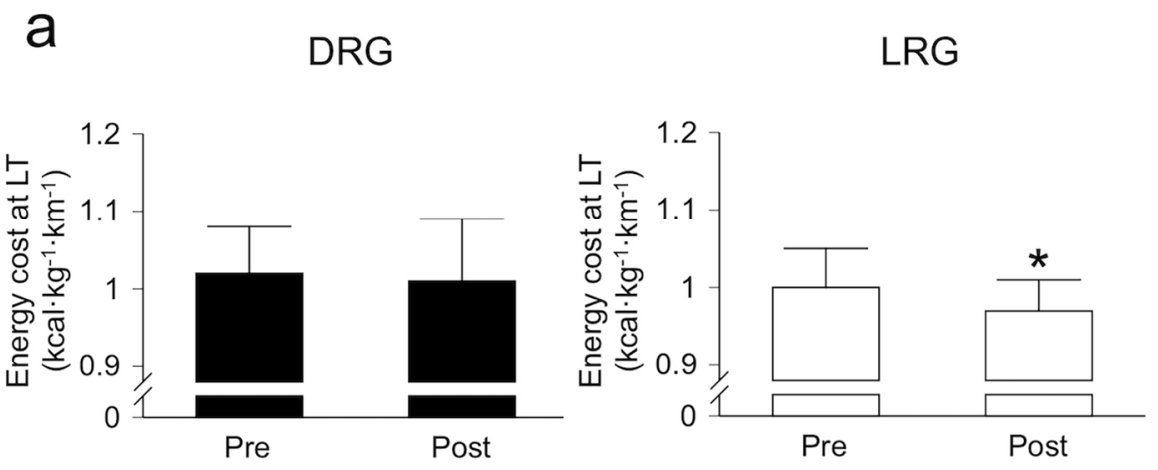

b
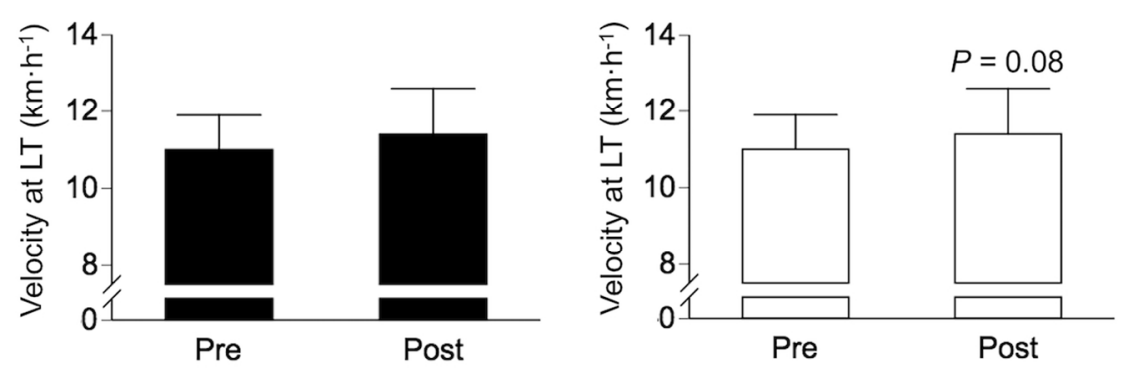

Figure 6. Energy cost at lactate threshold (LT) (a) and velocity at LT (b) before (pre) and after (post) the interventions. DRG: downhill running group (closed bars), LRG: level running group (open bars). Values are means \pm SDs. ${ }^{*}$ A significant change from pre at $P<0.05$.

$112 \times 150 \mathrm{~mm}(300 \times 300 \mathrm{DPI})$ 